\title{
"LA GESTIÓN ESTRATÉGICA Y SU RELACIÓN CON LA CAPTACIÓN DE ALUMNOS EN LA UNIVERSIDAD PRIVADA DE TACNA, PERIODO 2014- 2016"
}

\author{
"STRATEGIC MANAGEMENT AND ITS RELATIONSHIP WITH THE \\ COLLECTION OF STUDENTS AT THE PRIVATE UNIVERSITY OF TACNA, \\ PERIOD 2014-2016"
}

\section{Resumen}

La gestión estratégica de las instituciones de educación superior presenta un relativo bajo nivel de productividad; en tal sentido la pregunta fundamental que ha orientado a la investigación, en este campo, y que han tratado de responder los investigadores es: ¿cómo las organizaciones alcanzan y mantienen su ventaja competitiva? Esta investigación buscó demostrar que la gestión estratégica está relacionada con el incremento de la población estudiantil, medida a través de la captación de alumnos meta al cual se dirige la universidad. De otro lado, se ha utilizado información secundaria de la oficina de planificación de la Universidad Privada de Tacna. Se calculó el coeficiente de correlación de Pearson para analizar el grado de fuerza y el sentido de las variables relevantes de la gestión estratégica con la captación de alumnos a fin de comprobar la hipótesis principal, y para la comprobación de las hipótesis específicas planteadas se utilizó el test de diferencia de medias. Al realizar el contraste de la hipótesis principal, se encontró que la gestión estratégica de la Universidad Privada de Tacna está relacionada directa y significativamente con la captación de alumnos de su mercado meta. En el caso de las hipótesis específicas, no hay diferencias entre la planeación y la ejecución respecto a la difusión de la producción intelectual y los resultados de formación especializada.

PALABRAS CLAVE: Gestión estratégica, Instituciones educativas.
ASCENCIÓN AMÉRICO FLORES FLORES 1

\section{Abstract}

The strategic management of institutions of higher education presents a relatively low level of productivity; In this sense the fundamental question that has orientated the research in this field and that the researchers have tried to answer is: how do organizations reach and maintain their competitive advantage? This research seeks to demonstrate that strategic management is related to the increase of the student population, measured through the recruitment of students in the target market to which the university is directed. On the other hand, secondary information was used by the planning office of the Private University of Tacna. In addition, the Pearson correlation coefficient has been used to analyze the degree of strength and the meaning of the relevant variables of strategic management with the recruitment of students in order to verify the main hypothesis as well, the test of difference between two means was used to verify the specific hypotheses proposed.When contrasting the main hypothesis, it was found that the strategic management of the Private University of Tacna is directly and significantly related to the recruitment of students from its target. In the case of the specific hypotheses, there are no differences between planning and execution regarding the diffusion of intellectual production and the results of specialized training.

KEYWORDS: Strategic Management, Educational Institutions.

1 DOCTOR EN ADMINISTRACIÓN 


\section{INTRODUCCIÓN}

Actualmente, las instituciones de Educación Superior se ven enfrentadas a múltiples dificultades y retos (Tünnermann, 2003). El fenómeno de la globalización se ha convertido en un gran desafío para las instituciones de Educación Superior (Tsai \& Beverton, 2007). Adicionalmente, las instituciones de Educación Superior se debaten internamente, entre múltiples identidades (Arechavala, 2001; Henkel, 2005; Sporn, 1996) y los gestores y los directores administrativos intentan promover los valores y los objetivos asociados con la productividad, la eficiencia, la eficacia, la calidad, con el propósito de enfrentar los grandes retos que plantea el entorno, desde lo económico, lo tecnológico, lo político y lo social (Winter \& Sarros, 2002). Muchas Instituciones Educativas han adoptado algunas de las propuestas de gestión, transformando lo que ellas hacen y cómo se gobiernan (Castro \& Ion, 2011). En el contexto de la Educación Superior en América Latina, los distintos gobiernos han promovido, en las últimas dos décadas, cambios importantes en las políticas educativas, con el propósito de lograr un mayor control de los resultados educativos (Brunner, 2011). Los gobiernos, en la mayoría de los países, han introducido marcos regulatorios más exigentes, para el reconocimiento oficial de las Instituciones Educativas superiores, así como para el otorgamiento de la plena autonomía (Brunner, 2011; Welsh \& Metcalf, 2003). Aunque la planificación estratégica se ha utilizado en el sector empresarial, desde mediados de los años setenta, su incorporación en las instituciones educativas Superiores, como mecanismo de gestión, se produjo en la década de los años noventa (Álamo \& García, 2007). Para lograr tanto una inserción efectiva de estos modelos de gestión, aparecen algunos obstáculos: la falta de liderazgo institucional, las limitaciones de los mecanismos, la evaluación de la gestión académica; $\mathrm{y}$, de manera determinante, la resistencia y la falta de compromiso de los académicos (Welsh \& Metcalf, 2003).

La Universidad Peruana no viene respondiendo adecuadamente a las necesidades y expectativas de la sociedad actual, caracterizada por la incertidumbre, la complejidad, la competitividad, la globalización, la internacionalización, el cambio, etc. Actualmente se percibe que la universidad peruana viene desarrollando gestión académica y administrativa sin una adecuada planificación universitaria, esto no significa que las universidades dejen de elaborar sus planes operativos, planes estratégicos, y presupuestos; sin embargo, dichos instrumentos de gestión, resultan siendo formulados y aprobados formalmente con intenciones de cumplimiento; generándose una imagen de una Universidad carente de norte y de perspectiva hacia el futuro; a este estado de situación no está ajena la Universidad Privada de Tacna.

Schmelkes G; nos dice que el planeamiento estratégico surgió después de la segunda Guerra Mundial .Los orígenes del planeamiento estratégico, fue introducido por primera vez a mediados de 1950. Manes, (1999) refiere que el tipo de dirección que se llevaba a cabo en los niveles más altos de la organización no era la dirección operacional sino la estratégica, aquella que guía, direcciona y pone límites a todo proceso operacional. Según David, F. (1990) la planificación estratégica puede definirse como un enfoque objetivo y sistemático para la toma de decisiones en una organización. Kotler \& Bloom (1988) asume asimismo que la planeación estratégica es el proceso gerencial de desarrollar y mantener una dirección estratégica que pueda alinear las metas y recursos de la organización con sus oportunidades cambiantes de mercadeo. Harvard Business Shool, señaló la importancia de diseñar una estrategia corporativa cabal que influye todas las facetas de las aspiraciones incluyendo producción, finanzas y mercadeo.

Según Porter (1991) la primera etapa de la gerencia estratégica se denomina formulación estratégica y la segunda etapa se configura cuando las investigaciones permiten determinar la importancia de lo que se denomina configuración interna de la organización y se denominó según planeación de habilidades. La tercera etapa se dio en respuesta de cambios socio-políticos y tecnológicos, las organizaciones realizan grandes esfuerzos para dar una respuesta estratégica oportuna a lo que se denominó respuesta administrativa. Milla (2007) nos dice que la "planeación estratégica" nos ayuda a adquirir un concepto muy claro de nuestra organización, la cual a su vez hace posible la formulación de planes y actividades 
que nos llevan hacia los objetivos organizacionales.

La planeación y la gestión estratégica es un campo académico relativamente joven. No obstante, la planeación y la gestión estratégica, como campo disciplinar presenta muchos atributos de una disciplina inmadura: debilidad en los consensos y un relativo bajo nivel de productividad (Boyd et al., 2005). Las investigaciones, en planeación y gestión estratégica, son impulsados más por factores universales que por particulares (Boyd et al., 2005). Desde sus inicios, ha sido reconocido como un campo multidisciplinario, que ha tomado prestado y asimilado el trabajo y los conceptos propuestos por otras disciplinas académicas relacionadas, como la Economía, la
Sociología y la Psicología (Ramos \& Ruíz, 2004). La Investigación en el campo de la Planificación Estratégica presenta un relativo bajo nivel de productividad; sin embargo, tanto la creación como la utilización del conocimiento científico siguen los lineamientos normativos de la creación científica (Boyd et al., 2005). La pregunta fundamental que ha orientado a la investigación, en este campo, y que han tratado de responder los investigadores es: ¿cómo las organizaciones alcanzan y mantienen su ventaja competitiva? De acuerdo con Teece, Pisano, et al. (1997), los trabajos de investigación, en el campo de la planeación estratégica, se pueden agrupar desde dos enfoques fundamentales: el enfoque basado en el poder de las fuerzas del entorno; y el enfoque basado en la eficiencia de las organizaciones.

\section{OBJETIVOS}

1. Analizar si existen diferencias entre la planeación y los resultados de los proyectos de investigación, entre la planeación y los resultados de formación especializada de los docentes, entre la planeación de las publicaciones científicas de producción de los docentes en revistas indizadas tanto a nivel nacional e internacional y los resultados obtenidos.
2. Analizar si existen diferencias entre la planeación de la difusión de la producción intelectual de los docentes y los resultados obtenidos y evaluar si existen diferencias entre la planeación de la participación de docentes en programas de intercambio académico a nivel nacional e internacional y los resultados obtenidos en la Universidad.

\section{METODOLOGÍA}

\section{Tipo de Investigación}

Diseño no experimental, investigación descriptiva y correlacional de corte longitudinal. El presente trabajo de investigación se realizó en la Universidad Privada de Tacna, tomándose en consideración el comportamiento de un conjunto de indicadores de gestión e indicadores de resultado, durante un periodo de tiempo comprendido entre los años 2013 - 2016. La recolección de datos se efectuó a través de la recopilación de información secundaria mediante mediante fichas para recolección de datos, hardware personal y software estadístico en base Excel. Se utilizó e Coeficiente de correlación de Pearson y diferencia de medias mediante "t" student.

\section{RESULTADOS}

La población estudiantil del año 2016 respecto al año anterior ha crecido en $5.17 \%$, sin embargo, si comparamos con respecto a los años 2013 y 2014 , este crecimiento ha sido muy superior, representado el $10.62 \%$ y $12.21 \%$ respectivamente.

En los últimos tres años se ha venido incrementando recursos a la capacitación de sus docentes, sin embargo, no es suficiente ya que debería destinarse un monto mayor a este rubro, debido a que la capacitación docente le permitirá a la universidad contar con un capital humano de primer nivel, el mismo que le ayudará a posicionarse en el mercado de una manera más exitosa (Fig. 01). 


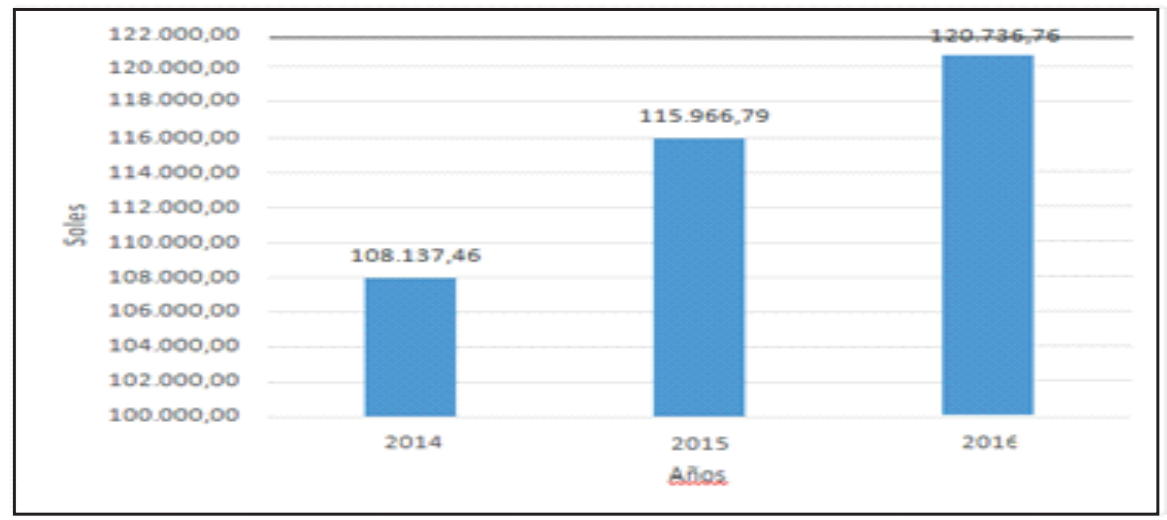

Fig. 01: Inversión en capacitación docente

La inversión en equipamiento que ejecuta la Universidad Privada de Tacna en los últimos dos años, es decir, 2015 y 2016 , se ha duplicado en el año 2016. La inversión en infraestructura ha crecido en $23,03 \%$ en el año 2016 respecto al año anterior. Así mismo, el financiamiento para esta inversión principalmente lo constituyen recursos ordinarios.

El presupuesto en publicidad ha crecido en $48.67 \%$ en el año 2016 respecto al año anterior.
La inversión en investigación, si bien ligeramente a aumentado, no va en relación a los objetivos que se buscan alcanzar por parte de la Universidad Privada de Tacna (Fig. 02). Es necesario precisar que hoy en día toda institución educativa debe potenciar este aspecto, el mismo que se ha constituido en la creación de una ventaja competitiva con relación a la competencia.

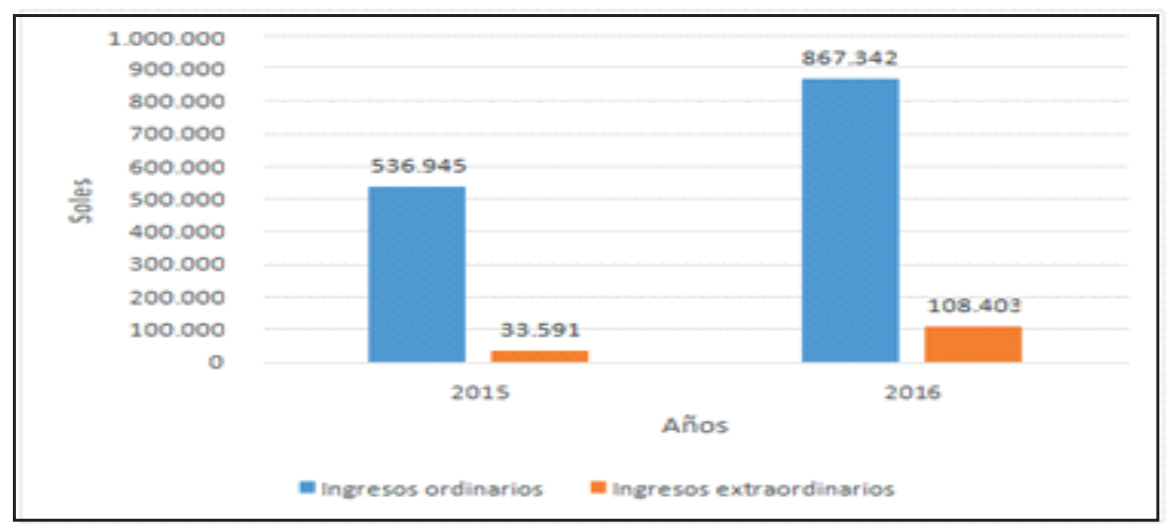

Fig. 02: Inversión en Investigación

Respecto al presupuesto de la Universidad Privada de Tacna, para el año 2015, se puede apreciar que la principal fuente de ingresos, lo constituye las pensiones de enseñanza de las seis facultades, lo que representa el $78.82 \%$ del total de los ingresos (Tabla 01), lo cual denota un gran riesgo debido a que no puede depender principalmente de un rubro, sino por el contrario, debería tener un mejor portafolio de ingresos, que pueden derivarse de otras actividades que puede ofrecer la universidad con su capital humano, así como su Know How.

Tabla 01: Presupuesto de Ingresos

\begin{tabular}{|l|r|c|}
\hline \multirow{2}{*}{ Conceptos } & \multicolumn{2}{c|}{2015} \\
\cline { 2 - 3 } & \multicolumn{1}{|c|}{ Total } & \% \\
\hline Ingresos & & \\
\hline PENSIONES & 27.720 .636 & $78,817 \%$ \\
\hline OTROS INGRESOS & 7.450 .147 & $21,183 \%$ \\
\hline Total Ingresos & $\mathbf{3 5 . 1 7 0 . 7 8 3}$ & $\mathbf{1 0 0 , 0 \%}$ \\
\hline
\end{tabular}


Con respecto al presupuesto de egresos para el año 2015, (Tabla 02) se puede apreciar que la principal salida de egresos, lo constituye el rubro de remuneraciones, el mismo que participa con el $62,64 \%$. Esto obedece a la naturaleza de una institución educativa, ya que esta brinda servicios.

Tabla 2: Presupuesto de Egreso

\begin{tabular}{|l|r|r|}
\hline \multicolumn{1}{|c|}{ CONCEPTOS } & \multicolumn{2}{|c|}{$\mathbf{2 0 1 5}$} \\
\hline Egresos & \multicolumn{1}{|c|}{ TOTAL } & \multicolumn{1}{c|}{$\%$} \\
\hline AMORTIZACIóN DE OBLIGACIONES & & \\
\hline Amortización Préstamos & 1.683 .614 & $4,79 \%$ \\
\hline & & \\
\hline INVERSIONES & 1.400 .000 & $3,98 \%$ \\
\hline REMUNERACIONES & $\mathbf{2 2 . 0 3 1 . 7 6 5}$ & $62,64 \%$ \\
\hline Remuneración Docentes & 12.674 .702 & $36,04 \%$ \\
\hline Remuneración Principal Administrativo & 4.034 .451 & $11,47 \%$ \\
\hline Subvenciones a Practicantes & 211.939 & $0,60 \%$ \\
\hline Gratificaciones & 2.362 .545 & $6,72 \%$ \\
\hline Seguridad Social & 1.550 .841 & $4,41 \%$ \\
\hline Seguro Social Practicantes & 21.120 & $0,06 \%$ \\
\hline Compensación por Tiempo de Servicios & 1.176 .167 & $3,34 \%$ \\
\hline Contingencias & 2.750 .000 & $7,82 \%$ \\
\hline Gastos de Servicios Prestados por Terceros & 5.200 .000 & $14,78 \%$ \\
\hline Gastos Tributarios & 51.907 & $0,15 \%$ \\
\hline Gastos Diversos de Gestión & 1.658 .409 & $4,72 \%$ \\
\hline Gastos Financieros & 395.089 & $1,12 \%$ \\
\hline Total Egresos & $\mathbf{3 5 . 1 7 0 . 7 8 3}$ & $\mathbf{1 0 0 , 0 0 \%}$ \\
\hline
\end{tabular}

El presupuesto de ingresos para el año 2016 (Tabla 03), las pensiones de enseñanza constituyen el $84,12 \%$ del total de los ingresos, tan igual que para el año 2015. Se puede considerar un alto riesgo, debiendo la universidad diversificar sus ingresos a través del desarrollo de otros servicios que puede ofrecer al mercado con los recursos con los que cuenta.

Tabla 3: Ingresos

\begin{tabular}{|c|c|c|}
\hline \multirow{2}{*}{ CONCEPTO } & \multicolumn{2}{|c|}{2016} \\
\hline & Total & $\%$ \\
\hline Ingresos & & \\
\hline PENSIONES & 34.020 .944 & $84,12 \%$ \\
\hline OTROS INGRESOS & 6.423 .490 & $15,88 \%$ \\
\hline Total Ingresos & 40.444 .434 & $100,00 \%$ \\
\hline
\end{tabular}

Observamos en la tabla 04 los egresos considerados para el año 2016. Al respecto, podemos indicar que el rubro de remuneraciones sigue siendo un aspecto importante, el cual representa el $61.91 \%$ del total del presupuesto de egresos, esto se justifica por el carácter de la institución, la misma que ofrece servici2os educativos. 
Tabla 04: Presupuesto de Egresos

\begin{tabular}{|l|r|r|}
\hline \multirow{2}{*}{ CONCEPTOS } & \multicolumn{2}{|c|}{$\mathbf{2 0 1 6}$} \\
\cline { 2 - 3 } & \multicolumn{1}{|c|}{ TOTAL } & \multicolumn{1}{c|}{$\%$} \\
\hline AMORTIZACIóN DE OBLIGACIONES & & \\
\hline Amortización Préstamos & 1.957 .798 & $4,84 \%$ \\
\hline INVERSIONES & 1.478 .000 & $3,65 \%$ \\
\hline GASTOS DE OPERACIóN & 39.258 .636 & $97,07 \%$ \\
\hline Gastos de Personal & & \\
\hline Remuneraciones & 25.039 .071 & $61,91 \%$ \\
\hline Remuneración Docentes & 12.917 .377 & $31,94 \%$ \\
\hline Remuneración Administrativo & 3.420 .228 & $8,46 \%$ \\
\hline Subvenciones a Practicantes & 249.067 & $0,62 \%$ \\
\hline Gratificaciones* & 3.025 .677 & $7,48 \%$ \\
\hline Seguridad Social & 1.709 .877 & $4,23 \%$ \\
\hline Compensación por Tiempo de Servicios & 1.209 .286 & $2,99 \%$ \\
\hline Asistencia y Subsidios & 2.507 .559 & $6,20 \%$ \\
\hline Contingencias & 2.250 .000 & $5,56 \%$ \\
\hline Planes de Mejora & 1.000 .000 & $2,47 \%$ \\
\hline Becas & 125.000 & $0,31 \%$ \\
\hline Contingencias & 1.125 .000 & $2,78 \%$ \\
\hline Gastos de Servicios Prestados por Terceros & 7.326 .000 & $18,11 \%$ \\
\hline Gastos Tributarios & 200.000 & $0,49 \%$ \\
\hline Gastos Diversos de Gestión & 2.103 .160 & $5,20 \%$ \\
\hline Gastos Financieros & 90.405 & $0,22 \%$ \\
\hline Total Egresos & 40.444 .434 & $100,00 \%$ \\
\hline & & \\
\hline
\end{tabular}

Respecto a personal docente nombrado, en términos generales, ha venido reduciéndose, producto de las jubilaciones o cese de personal principalmente por edad (Tabla 05).

Tabla 05: Docentes ordinarios por Facultad

\begin{tabular}{|l|c|c|c|c|}
\hline \multicolumn{1}{|c|}{ FACULTAD } & $\mathbf{2 0 1 3}$ & $\mathbf{2 0 1 4}$ & $\mathbf{2 0 1 5}$ & $\mathbf{2 0 1 6}$ \\
\hline Facultad de Derecho y Ciencias Políticas & & & & \\
\hline Principal & 7 & 6 & 6 & 3 \\
Asociado & 5 & 5 & 5 & 4 \\
Auxiliar & 3 & 3 & 3 & 3 \\
\hline Facultad de Educación, Cs. de la Comunicación & & & & \\
\hline y Humanidades & & & & \\
Principal & 7 & 6 & 6 & 5 \\
Asociado & 1 & 1 & 1 & 1 \\
Auxiliar & 2 & 2 & 2 & 2 \\
\hline Facultad de Ingeniería & & & & \\
\hline Principal & 8 & 8 & 7 & 6 \\
Asociado & 5 & 5 & 5 & 5 \\
Auxiliar & 3 & 3 & 3 & 3 \\
\hline Facultad de Cs. de la Salud & & & & \\
\hline Principal & 3 & 3 & 3 & 2 \\
Asociado & 6 & 6 & 6 & 6 \\
Auxiliar & 6 & 6 & 6 & 6 \\
\hline Facultad de Cs. Empresariales & & & & \\
\hline Principal & 5 & 5 & 5 & 5 \\
Asociado & 4 & 4 & 4 & 3 \\
Auxiliar & 0 & 0 & 0 & 0 \\
\hline Facultad de Arquitectura y Urbanismo & & & & \\
\hline Principal & 3 & 3 & 3 & 3 \\
Asociado & 4 & 4 & 4 & 4 \\
Auxiliar & 0 & 0 & 0 & 0 \\
\hline & 72 & 70 & 69 & 61 \\
\hline
\end{tabular}


Se puede analizar que la variable captación de alumnos por parte de la Universidad Privada de Tacna, se encuentra relacionada con la inversión en publicidad, la inversión en capacitación docente, la inversión en equipamiento, la inversión en infraestructura y la inversión en investigación (tabla 07). a un nivel de confianza del $95 \%$. Así mismo, se puede concluir que existe una relación positiva y significativa, es decir, ante un incremento de las variables genera un incremento en el nivel de la población estudiantil de la Universidad Privada de Tacna.

Tabla 06: Correlaciones

\begin{tabular}{|c|c|c|c|c|c|c|c|}
\hline & & $\begin{array}{l}\text { Pobla } \\
\text { ción }\end{array}$ & $\begin{array}{l}\text { Publici } \\
\text { dad }\end{array}$ & $\begin{array}{l}\text { Capacit } \\
\text { ación_- } \\
\text { Docente }\end{array}$ & $\begin{array}{l}\text { Inv_Equ } \\
\text { ipamiento }\end{array}$ & $\begin{array}{l}\text { Inv_Infr } \\
\text { aestruct } \\
\text { ura }\end{array}$ & $\begin{array}{l}\text { Inv_Inve } \\
\text { stigación }\end{array}$ \\
\hline Población & $\begin{array}{l}\text { Correlación de } \\
\text { Pearson } \\
\text { Sig. (bilateral) } \\
\text { N }\end{array}$ & 4 & $\begin{array}{r}, 998^{\star *} \\
, 002 \\
4 \\
\end{array}$ & $\begin{array}{r}, 951^{*} \\
, 049 \\
4\end{array}$ & $\begin{array}{r}, 953^{*} \\
, 047 \\
4 \\
\end{array}$ & $\begin{array}{r}, 971^{*} \\
, 029 \\
4 \\
\end{array}$ & $\begin{array}{r}, 967^{*} \\
\\
, 033 \\
4\end{array}$ \\
\hline Publicidad & $\begin{array}{l}\text { Correlación de } \\
\text { Pearson } \\
\text { Sig. (bilateral) } \\
\text { N }\end{array}$ & $\begin{array}{r}, 998^{* *} \\
, 002 \\
4\end{array}$ & 4 & $\begin{array}{r}, 941 \\
\\
, 059 \\
4 \\
\end{array}$ & $\begin{array}{r}, 948 \\
\\
, 052 \\
4 \\
\end{array}$ & $\begin{array}{r}, 963^{*} \\
, 037 \\
4 \\
\end{array}$ & $\begin{array}{r}, 971^{*} \\
, 029 \\
4\end{array}$ \\
\hline $\begin{array}{l}\text { Capacitación } \\
\text { Docente }\end{array}$ & $\begin{array}{l}\text { Correlación de } \\
\text { Pearson } \\
\text { Sig. (bilateral) } \\
\text { N }\end{array}$ & $\begin{array}{r}, 951^{*} \\
, 049 \\
4 \\
\end{array}$ & $\begin{array}{r}, 941 \\
\\
, 059 \\
4 \\
\end{array}$ & 4 & $\begin{array}{r}, 997^{* *} \\
, 003 \\
4 \\
\end{array}$ & $\begin{array}{r}, 997^{* *} \\
, 003 \\
4 \\
\end{array}$ & $\begin{array}{r}, 841 \\
\\
, 159 \\
4 \\
\end{array}$ \\
\hline Inv_Equipami & $\begin{array}{l}\text { Correlación de ento } \\
\text { Pearson } \\
\text { Sig. (bilateral) } \\
\text { N }\end{array}$ & $\begin{array}{r}, 953^{*} \\
, 047 \\
4 \\
\end{array}$ & $\begin{array}{r}, 948 \\
\\
, 052 \\
4 \\
\end{array}$ & $\begin{array}{r}, 997^{\star \star} \\
, 003 \\
4 \\
\end{array}$ & 4 & $\begin{array}{r}, 997^{* *} \\
, 003 \\
4 \\
\end{array}$ & $\begin{array}{r}, 847 \\
\\
, 153 \\
4 \\
\end{array}$ \\
\hline $\begin{array}{l}\text { Inv_Infraestru } \\
\text { ctura }\end{array}$ & $\begin{array}{l}\text { Correlación de } \\
\text { Pearson } \\
\text { Sig. (bilateral) } \\
\text { N }\end{array}$ & $\begin{array}{r}, 971^{*} \\
, 029 \\
4 \\
\end{array}$ & $\begin{array}{r}, 963^{*} \\
, 037 \\
4\end{array}$ & $\begin{array}{r}, 997^{\star *} \\
, 003 \\
4\end{array}$ & $\begin{array}{r}, 997^{* *} \\
, 003 \\
4\end{array}$ & 4 & $\begin{array}{r}, 877 \\
\\
, 123 \\
4\end{array}$ \\
\hline Inv_Investiga & $\begin{array}{l}\text { Correlación de ción } \\
\text { Pearson } \\
\text { Sig. (bilateral) } \\
\text { N }\end{array}$ & $\begin{array}{r}, 967^{\star} \\
, 033 \\
4\end{array}$ & $\begin{array}{r}, 971^{*} \\
, 029 \\
4\end{array}$ & $\begin{array}{r}, 841 \\
4,159 \\
4\end{array}$ & $\begin{array}{r}, 847 \\
\\
, 153 \\
4 \\
\end{array}$ & $\begin{array}{r}, 877 \\
, 123 \\
4 \\
\end{array}$ & 4 \\
\hline
\end{tabular}

No existe diferencia significativa en el número de proyectos presentados por los alumnos dentro de las líneas de investigación planteadas por la Universidad Privada de Tacna y la meta propuesta en la etapa de planificación en los últimos tres años. Existe diferencia significativa entre la planeación y los resultados de formación especializada de los docentes que demandan los programas académicos de la universidad y la meta propuesta en la etapa de planificación en los últimos tres años ( $p>0.05$ ). No existe diferencia significativa entre las publicaciones científicas de la producción de los docentes en revistas indizadas tanto a nivel nacional como internacional y la meta propuesta en la etapa de planificación en los últimos tres años ( $p>0.05)$.

Existe diferencia significativa entre la planeación y los resultados de formación especializada de los docentes, así como los resultados de la participación de los docentes en programas de intercambio académico de la universidad que demandan los programas académicos de la universidad y la meta propuesta en la etapa de planificación en los últimos tres años $(p<0.05)$

\section{DISCUSIÓN}

Se pude concluir que la gestión estratégica que viene desarrollando la Universidad Privada de Tacna, a través de la inversión en capacitación docente, inversión en infraestructura y equipamiento, así como, investigación científica y publicidad, están relacionadas con el crecimiento de la población estudiantil medida a través de una mayor captación de alumnos, lo cual se ha demostrado a través del coeficiente de correlación de Pearson, dándonos a conocer que 
existe además una relación positiva entre ellas.

Con relación a si existen diferencias entre lo planificado y ejecutado, respecto a los proyectos presentados por los estudiantes en relación a las líneas de investigación priorizadas, publicaciones científicas de los docentes en revistas indizadas y la participación de los docentes en programas de intercambio académico, se pude concluir que existen diferencias entre lo planificado y ejecutado. Respecto a si existen diferencias entre lo planificado y ejecutado, con relación a la difusión de producción intelectual de los docentes y los resultados de formación especializada de los mismos, se encontró que no existen diferencias, en consecuencia se ha logrado alcanzar la meta planificada por la Universidad. La población total estudiantil de la Universidad Privada de Tacna ha tenido una evolución creciente y constante durante los años 2013 al 2016 . Este crecimiento se debe al desarrollo de un mejor nivel académico y a la inversión directa en el mejoramiento de la infraestructura y el equipamiento, así como a una adecuada estrategia publicitaria sustentada en sus fortalezas. Las facultades en general han mantenido un crecimiento sostenido, sobre la base de estrategias internas, así como la evolución del entorno. Respecto al primer caso se puede mencionar el desarrollo de nuevos productos, con lo cual ha ampliado su portafolio, además del proceso de acreditación en el cual se encuentra inmerso hace tres años con la CNA de Colombia. Dentro del aspecto externo, se puede mencionar el incremento de la inversión pública en los últimos años principalmente en el desarrollo de infraestructura, lo cual se ha traducido en una mayor demanda por las carreras ligadas a la construcción. En relación a la evolución de los recursos destinados a la inversión en capacitación docente, se puede indicar que si bien es cierto ha habido un crecimiento en los últimos cuatro años, es decir 2013 al 2016, estos son insuficientes, ya que debería destinarse un presupuesto mayor para este rubro, ya que los docente son la piedra angular de los servicios educativos y por efectos multiplicadores que esto generaría. La inversión en equipamiento también ha tenido un crecimiento en los últimos años, pero este no es muy significativo, es necesario precisar que se debería destinar un mayor monto a este aspecto, dado el avance de la tecnología que obliga sobre todo a las instituciones educativas a contar con equipamiento moderno, a fin de garantizar adecuado proceso de enseñanza aprendizaje que garantiza una educación de calidad. La inversión en infraestructura en los últimos años no ha sido significativa de acuerdo a las necesidades de la casa superior de estudios, sin embargo, es necesario precisar que el presente año 2017 se está destinando 50 millones de soles con financiamiento de terceros para el desarrollo de infraestructura moderna de las facultades de ingeniería y arquitectura, lo cual apunta a brindar un mejor servicio educativo y por ende a generar una mayor demanda por los productos que se ofertan. Un aspecto relevante en toda organización educativa lo constituye la investigación, sin embargo, los recursos destinados a este rubro han sido demasiado bajos, por lo que no se promueve el desarrollo de actividades que apunten a fortalecer el desarrollo de productos de investigación que a largo plazo van a contribuir con el posicionamiento de la universidad en su mercado meta.

Se debe mejorar el equipamiento y la infraestructura, con la finalidad de brindar un mejor servicio a sus clientes actuales y potenciales, lo cual redundará en una mejora en el proceso de enseñanza aprendizaje al contar con la tecnología moderna para la educación, así como ambientes adecuados. Es necesario precisar que los alumnos son nuestros clientes y constituyen nuestra mejor estrategia de marketing de nuestros productos educativos. Respecto a las diferencias que existen entre lo planificado y ejecutado con relación a la investigación desarrollada por los alumnos y las publicaciones de los docentes en revistas indizadas, así como la participación de estos últimos en el intercambio académico, se debe fortalecer y promover sosteniblemente en el tiempo el desarrollo de investigación priorizando esta actividad en sus planes de trabajo, con la finalidad de desarrollar y publicar sus investigaciones tanto a nivel nacional como internacional. De otro lado, esto permitirá que los docentes puedan presentar sus publicaciones en congresos, tanto a nivel nacional como internacional, posicionando de esta manera la universidad en el mercado global. Respecto a las actividades planificadas y cumplidas se debe seguir motivando y propiciando la difusión intelectual de los docentes no solamente a nivel local o nacional, sino a nivel internacional y continuar con el desarrollo de especializaciones a fin de contar con los recursos humanos adecuados para el proceso de enseñanza aprendizaje de acuerdo a los avances del 
conocimiento científico para cada uno de los productos que oferta la universidad actualmente y el futuro. La Universidad Privada de Tacna, debe implementar un conjunto de estrategias orientadas hacia el mercado (clientes) que le permitan captar una mayor cuota del mercado meta al cual se dirige, tales como, el mejoramiento de los docentes a través del fortalecimiento de programas de capacitación en el extranjero, sobre todo en universidades de mayor prestigio del mundo, lo cual redundaría en elevar el nivel académico y por ende generar efectos multiplicadores tanto internos como externos en el mercado actual y potencial. Un aspecto fundamental que debe impulsarse en forma inmediata y hacerla sostenible en el largo plazo, viene a ser el desarrollo de actividades de investigación, a través de estrategias que impulsen la participación de alumnos, docentes de la universidad y docentes de otras universidad del extranjero a través del desarrollo de investigación conjuntas, esto redundará en el posicionamiento de la universidad, así como la obtención de recursos de la empresa privada y pública, como extranjera, para el desarrollo de esta actividad relevante para el sector de educación universitaria. Dado que los recursos económicos destinados a actividades de publicidad no son tan importantes, se debe optimizar los mismos utilizando los medios que están dirigidos a nuestro mercado meta, de tal manera que se pueda optimizar los recursos escasos. Sin embargo, es necesario tener en consideración que elevando el nivel académico y contando con el equipamiento y la infraestructura moderna, así como la acreditación, nos permitirá hacer de cada uno de los alumnos un agente de publicidad y promoción de la universidad. Respecto a la selección de recursos humanos para la universidad, específicamente docentes, se debe seleccionar adecuadamente de acuerdo a las carreras profesionales que se ofertan y sobre todo al perfil que debe desarrollar la universidad conjuntamente con las facultades, enfatizando la investigación como estrategia medular que dará sus frutos en el largo plazo. Se debe propiciar e implementar un conjunto de estrategia traducidas en acciones de corto, mediano y largo plazo con la finalidad de contar con un capital humano destinado a la investigación, para lo cual se debe capacitar en las mejores universidades del Perú o preferentemente en el extranjero a los docentes de las diferentes facultades y especialidades a fin de desarrollar investigaciones científicas que puedan ser publicadas en revistas internacionales de alto impacto, lo cual se complementaría con estrategias de motivación económica u de otro tipo a fin de seguir incentivando al docente investigador. El presente trabajo de investigación destaca la importancia de la gestión estratégica como instrumento técnico para el fortalecimiento de la gestión institucional en el marco de un plan estratégico institucional, para ello, se requiere que los procesos de planeación, organización, dirección y control en toda organización sean estratégicos.

Presentado: Marzo 2017

Aceptado: Junio 2017

\section{REFERENCIAS BIBLIOGRÁFICAS}

Álamo, F., \& García, M. (2007). El proceso estratégico en el sector público: Análisis en el contexto de las universidades Españolas. Investigaciones Europeas de Dirección y Economía de las Empresas.

Arechavala, R. (2001). El Gobierno de las Universidades Públicas: ¿Es posible comprender un sistema caótico? Revista de la Educación Superior - ANUIES, 118. México

Boyd, B., Finkelstein, S., \& Gove, S. (2005). How advanced is the strategy paradigm? The role of particularism and universalism in shaping research outcomes. Strategic Management Journal, 26(9), 14.

Brunner, J. J. (2011). Gobernanza Universitaria: tipología, dinámicas y tendencias. Revista de Educación. Ministerio de Educación - Gobierno de España.
Castro, D., \& Ion, G. (2011). Dilemas en el gobierno de las universidades españolas: autonomía, estructura, participación y desconcentración. Revista de Educación. Ministerio de Educación - Gobierno de España.

David, F. (1990). La gerencia estratégica. Colombia: Legis

Henkel, M. (2005). Academic identity and autonomy in a changing policy environment. Journal of Higher Education, 49, 155 - 176.

Kotler, P. y Bloom, P. (1988). Mercadeo de servicios profesionales. Bogotá: Legis.

Manes, J. (1999). Gestión estratégica para instituciones educativas. Guía para planificar estrategias de gerenciamiento institucional. Argentina: Granica.

Milla L. (2007) El Poder del Planeamiento Estratégico. Perú: San Marcos E.I.R.L. 
Porter, M. (1991). Towards a Dynamic Theory of Strategy. Strategic Management Journal, 12, 23.

Ramos, A., \& Ruíz, J. (2004). Changes in the intellectual structure of strategic management research: a bibliometric study of the Strategic Management Journal, 1980-2000. Strategic Management Journal, 25(10), 24.

Schmelkes, S. (1996). Calidad de la educación y gestión escolar. México: Secretaria de Educación Pública.

Sporn, B. (1996). Managing university culture: an analysis of relationship between institutional culture and management approaches. Higher Education, 32(1), 41-61

Teece, D., Pisano, G., \& Shuen, A. (1997). Dynamic capabilities and strategic management. Strategic Management Journal, 18(7).
Tsai, Y., \& Beverton, S. (2007). Top-down management: an effective tool in higher education?. International Journal of Educational Management, 21(1), 6-16.

Tünnermann, C. (2003). La Universidad Latinoamericana ante los retos del siglo XXI. México, D.F.: Unión de Universidades de América Latina - UDUAL.

Welsh, J. F., \& Metcalf, J. (2003). Faculty and Administrative Support for Institutional Effectiveness Activities: A Bridge accross the Chasm? The Journal of Higher Education, 74(4), 445-468

Winter, R. P., \& Sarros, J. C. (2002). Corporate reforms to Australin universities: Views from the academic heartland. Journal of Institutional Research, 11(2), 92 - 104. 